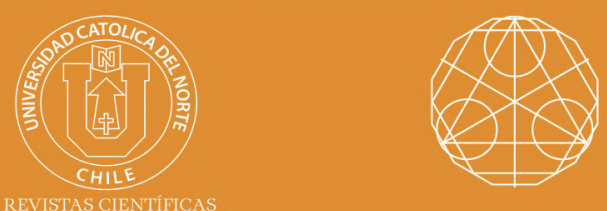

\title{
Upper triangular operator matrices and limit points of the essential spectrum
}

M. Karmouni*

A. Tajmouati* (iD orcid.org/0000-0003-1572-1241

A. El Bakkali***

*Cadi Ayyad University, Multidisciplinary Faculty, Safí, Morocco.

$\checkmark$ mohammed.karmouni@ucama

${ }^{* *}$ Sidi Mohamed Ben Abdellah Univerșity, Faculty of Sciences Dhar Al Mahraz, LAMA, Fez, Morocco.

Mabdelaziz.tajmouati@usmba.ac.ma

${ }^{* * *}$ Chouaib Doukkali University, Faculty of Science, Dept. of Mathematics, Elijadida, Morocco.

$\checkmark$ abdeslamelbakkalii@gmail.com

Received: November 2017 | Accepted: May 2019

\section{Abstract:}

In this paper, we investigate the limit points set of essential spectrum of upper triangular operator matrices

$$
M_{c}\left(\begin{array}{ll}
A & C \\
0 & B
\end{array}\right)
$$

We prove that acco ${ }_{e}\left(M_{c}\right) \cup W=\operatorname{acco}_{e}(A) \cup \operatorname{acc\sigma }_{e}(B)$ where Wis the union of certain holes in accoe $\left(M_{C}^{e}\right)$, which happen to be subsets of acco $\sigma_{e}(B) \cap \operatorname{acco}_{e}(A)$. Also, several sufficient conditions for

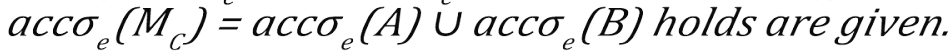

Keywords: Fredholm operator; Essential spectra; Limit point; Operator matrices.

MSC (2000): 47A10, 47A11.

\section{Cite this article as (IEEE citation style):}

M. Karmouni, A. Tajmouati andA. El Bakkali, "Upper triangular operator matrices and limit points of the essential spectrum", Proyecciones (Antofagasta, On line), vol. 38, no. 3, pp. 401-409, Aug. 2019, doi: 10.22199/issn.0717-6279-201903-0026. [Accessed dd-mm-yyyy].

Article copyright: (C) 2019 M. Karmouni, A. Tajmouati and A. El Bakkali. This is an open access article distributed under the terms of the Creative Commons Licence, which permits unrestricted use and distribution provided the original author and source are credited. 


\section{Introduction and Preliminaries}

Let $X, Y$ be infinite dimensional complex Banach spaces and $\mathcal{B}(X, Y)$ denote the complex algebra of all bounded linear operators from $X$ to $Y$. For $Y=X$ we write $\mathcal{B}(X, X)=\mathcal{B}(X)$. If $T \in \mathcal{B}(X)$, we denote by $T^{*}, N(T)$, $R(T), \sigma_{a p}(T), \sigma_{s u}(T), \sigma(T)$, respectively the adjoint, the null space, the range, the approximate point spectrum, the surjectivity spectrum and the spectrum of $T$.

A bounded linear operator is called an upper semi-Fredholm (resp, lower semi-Fredholm) if $\alpha(T)=\operatorname{dim} N(T)<\infty$ and $R(T)$ is closed (resp, $\beta(T)=\operatorname{codim} R(T)<\infty)$. $T$ is semi-Fredholm if it is a lower or upper semiFredholm operator. The index of a semi Fredholm operator $T$ is defined by $\operatorname{ind}(T)=\alpha(T)-\beta(T)$.

$T$ is a Fredholm operator if is a lower and upper semi-Fredholm operator. The essential spectrum of $T$ is the subset of $\mathbf{C}$ defined by:

$$
\sigma_{e}(T)=\{\lambda \in \mathbf{C}: T-\lambda I \text { is not a Fredholm operator }\}
$$

Let $T \in \mathcal{B}(X, Y), T$ is said to be left Atkinson if $T$ is upper semiFredholm and $R(T)$ is complemented in $X$, and it is said to be right Atkinson if $T$ is lower semi-Fredholm and $N(T)$ is complemented in $X$ (see [1]). The left and right Atkinson spectra are the subsets of $\mathbf{C}$ defined respectively by:

$$
\begin{array}{r}
\sigma_{l e}(T)=\{\lambda \in \mathbf{C}: T-\lambda I \text { is not a left Atkinson operator }\} \\
\sigma_{r e}(T)=\{\lambda \in \mathbf{C}: T-\lambda I \text { is not a right Atkinson operator }\} \\
\sigma_{e}(T), \sigma_{r e}(T) \text { and } \sigma_{l e}(T) \text { are compact subset and we have }
\end{array}
$$

$$
\sigma_{e}(T)=\sigma_{r e}(T) \cup \sigma_{l e}(T)
$$

For $A \in \mathcal{B}(X), B \in \mathcal{B}(Y)$, we denote by $M_{C} \in \mathcal{B}(X \oplus Y)$ the operator matrix acting on the product of Banach space $X \oplus Y$ [5]:

$$
M_{C}=\left(\begin{array}{cc}
A & C \\
0 & B
\end{array}\right)
$$

It is well know that, in the case of infinite dimensional, the inclusion $\sigma\left(M_{C}\right) \subset \sigma(A) \cup \sigma(B)$, may be strict. This motivates serval authors to study the defect $\left(\sigma_{*}(A) \cup \sigma_{*}(B)\right) \backslash \sigma_{*}\left(M_{C}\right)$ where $\sigma_{*}$ runs different type of spectra. 
If $H$ and $K$ are Hilbert spaces, Du and Pan [5] have studied the description of $\bigcap_{C \in \mathcal{B}(K, H)} \sigma\left(M_{C}\right)$ by showing that

$$
\bigcap_{C \in \mathcal{B}(K, H)} \sigma\left(M_{C}\right)=\sigma_{a p}(A) \cup \sigma_{s u}(B) \cup\{\lambda \in \mathbf{C}: \alpha(B-\lambda) \neq \beta(A-\lambda)\}
$$

Han H.Y. Lee and W. Y. Lee [6] extended the result to the Banach spaces. In [3], D.S. Djordjevic give a description of $\bigcap_{C \in \mathcal{B}(K, H)} \sigma_{e}\left(M_{C}\right)$, he showed the following theorem.

Theorem $1.1(3)$. . For given $(A, B) \in \mathcal{B}(X) \times \mathcal{B}(Y)$ the following holds:

$$
\bigcap_{C \in \mathcal{B}(Y, X)} \sigma_{e}\left(M_{C}\right)=\sigma_{l e}(A) \cup \sigma_{r e}(B) \cup W(A, B)
$$

Where $W(A, B)=\{\lambda \in \mathbf{C}, N(B-\lambda)$ and $X / \overline{R(A-\lambda)}$ are not isomorphic up to a finite dimensional subspace $\}$

In [9], the authors showed the following theorem.

Theorem $1.2(9)$. Let $(A, B) \in \mathcal{B}(X) \times \mathcal{B}(Y)$ and $C \in \mathcal{B}(Y, X)$. Then

$$
\sigma_{e}\left(M_{C}\right) \cup W_{e}=\sigma_{e}(A) \cup \sigma_{e}(B)
$$

where $W_{e}$ is the union of certain holes in $\sigma_{e}\left(M_{C}\right)$, which happen to be subsets of $\sigma_{e}(A) \cap \sigma_{e}(B)$.

For a compact subset $K$ of $\mathbf{C}$, let $a c c K$, int $K$, iso $K, \partial K$ and $\eta(K)$ be the set of all points of accumulation of $K$, the interior of $K$, the isolated points of $K$, the boundary of $K$ and the polynomially convex hull of $K$ respectively.

In this paper, we investigate the relationship between $a c c \sigma_{e}\left(M_{C}\right)$ and $a c c \sigma_{e}(A) \cup a c c \sigma_{e}(B)$. We show that the passage from $a c c \sigma_{e}\left(M_{0}\right)$ to $a c c \sigma_{e}\left(M_{C}\right)$ can be described as follows:

$$
\operatorname{acc}_{e}\left(M_{C}\right) \cup W=\operatorname{acc} \sigma_{e}\left(M_{0}\right)=a c c \sigma_{e}(A) \cup a c c \sigma_{e}(B)
$$

where $W$ is the union of certain holes in $a c c \sigma_{e}\left(M_{C}\right)$, which happen to be subsets of $a \operatorname{ccc} \sigma_{e}(A) \cap \operatorname{acc}_{e}(B)$. 


\section{Main results}

We start this section by proving that the limit point of essential spectrum set of a direct sum is the limit point of essential spectra of its summands.

Proposition 2.1. Let $(A, B) \in \mathcal{B}(X) \times \mathcal{B}(Y)$ and $C \in \mathcal{B}(Y, X)$. Then

$$
\operatorname{acc}_{e}\left(M_{0}\right)=a c c \sigma_{e}(A) \cup a c c \sigma_{e}(B)
$$

Proof. We have $\lambda \in \operatorname{acc} \sigma_{e}\left(M_{0}\right)$ if and only if $\lambda \in \operatorname{acc}\left(\sigma_{e}(A) \cup \sigma_{e}(B)\right)=$ $\operatorname{acc}\left(\sigma_{e}(A)\right) \cup \operatorname{acc}\left(\sigma_{e}(B)\right)$.

Lemma 2.1. Let $(A, B) \in \mathcal{B}(X) \times \mathcal{B}(Y)$ and $C \in \mathcal{B}(Y, X)$. Then

$$
a c c \sigma_{e}\left(M_{C}\right) \subseteq a c c \sigma_{e}\left(M_{0}\right)=a c c \sigma_{e}(A) \cup a c c \sigma_{e}(B)
$$

Proof. Without loss of generality, let $\lambda=0 \notin a c c \sigma_{e}(A) \cup a c c \sigma_{e}(B)$, then there exists $\varepsilon>0$ such that for any $\lambda, 0<|\lambda|<\varepsilon$, we have $A-\lambda I$ and $B-\lambda I$ are Fredholm. According to [4, Lemma 2.1], we have $M_{C}-\lambda I$ is Fredholm for any $\lambda, 0<|\lambda|<\varepsilon$, thus $0 \notin a c c\left(\sigma_{e}\left(M_{C}\right)\right)$. Therefore $a c c \sigma_{e}\left(M_{C}\right) \subseteq a c c \sigma_{e}(A) \cup a c c \sigma_{e}(B)$.

Definition 2.1. Let $T \in \mathcal{B}(X)$. We said that $T$ has the property aE at $\lambda \in \mathbf{C}$ if $\lambda \notin a c c \sigma_{e}(T)$.

The following lemma will be needed in the sequel.

Lemma 2.2. If two of $M_{C}, A$ and $B$ have the property $a E$ at 0 , then the third is also has the property $a E$.

Proof. $\quad i$ ) If $A$ and $B$ have the property $a E$, by lemma $2.1 M_{C}$ has the property $a E$.

ii) If $M_{C}$ and $A$ have the property $a E$, then $0 \notin a c c\left(\sigma_{e}\left(M_{C}\right)\right)$ and $0 \notin$ $\operatorname{acc}\left(\sigma_{e}(A)\right)$, thus there exists $\varepsilon>0$ such that $M_{C}-\lambda I$ and $A-\lambda I$ are Fredholm for every $\lambda, 0<|\lambda|<\varepsilon$. From [6, Corollary 5], $B-\lambda I$ is Fredholm for every $\lambda, 0<|\lambda|<\varepsilon$.

iii) If $B$ and $M_{C}$ have the property $a E$, the proof is similar to $i i$ ).

The first main result of this paper is the following theorem. 
Theorem 2.1. Let $(A, B) \in \mathcal{B}(X) \times \mathcal{B}(Y)$ and $C \in \mathcal{B}(Y, X)$. Then

$$
\operatorname{acc} \sigma_{e}\left(M_{C}\right) \cup W=a c c \sigma_{e}(A) \cup a c c \sigma_{e}(B)
$$

where $W$ is the union of certain holes in $\operatorname{acc} \sigma_{e}\left(M_{C}\right)$, which happen to be subsets of $\operatorname{acc}_{e}(B) \cap \operatorname{acc} \sigma_{e}(A)$.

Proof. We first claim that, for every $C \in \mathcal{B}(Y, X)$ we have

$$
\left(a c c \sigma_{e}(A) \cup a c c \sigma_{e}(B)\right) \backslash a c c \sigma_{e}(A) \cap a c c \sigma_{e}(B) \subseteq \operatorname{acc} \sigma_{e}\left(M_{C}\right)
$$

Indeed, let $\lambda \in\left(\operatorname{acco}_{e}(A) \cup a c c \sigma_{e}(B)\right) \backslash a c c \sigma_{e}(A) \cap a c c \sigma_{e}(B)$, then $\lambda \in \operatorname{acc}_{e}(A) \backslash a c c \sigma_{e}(B)$ or $\lambda \in a c c \sigma_{e}(B) \backslash a c c \sigma_{e}(A)$.

i) If $\lambda \in a c c \sigma_{e}(A) \backslash a c c \sigma_{e}(B)$, then $A$ has not the property $a E$ at $\lambda$ and $B$ has the property $a E$ at $\lambda$. Suppose that $\lambda \notin a c c \sigma_{e}\left(M_{C}\right)$, hence $M_{C}$ has the property $a E$ at $\lambda$, by lemma $2.2 A$ has the property $a E$ at $\lambda$, contradiction. So $\lambda \in \operatorname{acc} \sigma_{e}\left(M_{C}\right)$.

ii) If $\lambda \in a c c \sigma_{e}(B) \backslash a c c \sigma_{e}(A)$, by the same argument of $\left.i\right)$ we have $\lambda \in \operatorname{acco}_{e}\left(M_{C}\right)$.

Next, we claim that for every $C \in \mathcal{B}(Y, X)$ we have

$$
\eta\left(\operatorname{acco}_{e}\left(M_{C}\right)\right)=\eta\left(\operatorname{acc\sigma }_{e}(A) \cup \operatorname{acc} \sigma_{e}(B)\right)
$$

Since $a c c \sigma_{e}\left(M_{C}\right) \subseteq a c c \sigma_{e}(A) \cup a c c \sigma_{e}(B)$, we need to prove $\partial\left(a c c \sigma_{e}(A) \cup\right.$ $\left.\operatorname{acc}_{e}(B)\right) \subseteq \partial a c c \sigma_{e}\left(M_{C}\right)$. But since $\operatorname{int}\left(\operatorname{acc} \sigma_{e}\left(M_{C}\right)\right) \subseteq \operatorname{int}\left(\operatorname{acc} \sigma_{e}(A) \cup\right.$ $\left.a c c \sigma_{e}(B)\right)$, by the maximum modules theorem, it suffices to show that $\partial\left(a c c \sigma_{e}(A) \cup a c c \sigma_{e}(B)\right) \subseteq a c c \sigma_{e}\left(M_{C}\right)$. Without loss of generality, suppose $0 \in \partial\left(a c c \sigma_{e}(A) \cup a c c \sigma_{e}(B)\right)$. There are two cases to consider.

Case 1: If $0 \in \operatorname{acc}\left(\partial\left(\operatorname{acc} \sigma_{e}(A) \cup a c c \sigma_{e}(B)\right)\right)$, then there exists $\left(\lambda_{n}\right) \subseteq$ $\partial\left(\operatorname{acc}_{e}(A) \cup a c c \sigma_{e}(B)\right)$ such that $\lim _{n \rightarrow \infty} \lambda_{n}=0$, since

$$
\partial\left(\operatorname{acc}_{e}(A)\right) \subseteq \partial\left(\sigma_{e}(A)\right) \subseteq \sigma_{l e}(A) \subseteq \sigma_{e}\left(M_{C}\right)
$$

and

$$
\partial\left(\operatorname{acc}_{e}(B)\right) \subseteq \partial\left(\sigma_{e}(B)\right) \subseteq \sigma_{r e}(B) \subseteq \sigma_{e}\left(M_{C}\right)
$$


we have, $\lambda_{n} \in \sigma_{e}\left(M_{C}\right), n=1,2, \ldots$, hence $0 \in \operatorname{acc}\left(\sigma_{e}\left(M_{C}\right)\right)$.

Case 2: If $0 \in i s o\left(\partial\left(a c c \sigma_{e}(A) \cup a c c \sigma_{e}(B)\right)\right)$, since $a c c \sigma_{e}(A) \cup a c c \sigma_{e}(B)$ is closed, then $i s o\left(\partial\left(\operatorname{acc}_{e}(A) \cup a c c \sigma_{e}(B)\right)\right)=i s o\left(a c c \sigma_{e}(A) \cup a c c \sigma_{e}(B)\right)$.

$0 \in i \operatorname{so}\left(a c c \sigma_{e}(A) \cup a c c \sigma_{e}(B)\right)$, thus there exists $\varepsilon>0$ such that $\lambda \notin$ $\operatorname{acc}\left(\operatorname{acc} \sigma_{e}(A) \cup a c c \sigma_{e}(B)\right)$ for every $\lambda, 0<|\lambda|<\varepsilon$. Since $0 \in \operatorname{acc} \sigma_{e}(A) \cup$ $a c c \sigma_{e}(B)=\operatorname{acc}\left(\sigma_{e}(A) \cup \sigma_{e}(B)\right)$, there exists $\left(\mu_{n}\right) \subseteq \sigma_{e}(A) \cup \sigma_{e}(B)$ such that $\lim _{n \rightarrow \infty} \mu_{n}=0, \mu_{n} \neq 0$ for all $n$, thus there exists certain positive integer $N$ such that $0<\left|\mu_{n}\right|<\varepsilon$ for any $n \geq N$. Let $\lambda_{n}=\mu_{N+1+n}$, then $\lambda_{n} \in i \operatorname{sio}\left(\sigma_{e}(A) \cup \sigma_{e}(B)\right), n=1,2, .$. and $\lim _{n \rightarrow \infty} \lambda_{n}=0$. Since $\sigma_{e}(A)$ and $\sigma_{e}(B)$ are closed, then

$$
\begin{aligned}
i s o\left(\sigma_{e}(A) \cup \sigma_{e}(B)\right) & \subseteq i s o\left(\sigma_{e}(A)\right) \cup i s o\left(\sigma_{e}(B)\right) \\
& \subseteq \partial \sigma_{e}(A) \cup \partial \sigma_{e}(B) \\
& \subseteq \sigma_{l e}(A) \cup \sigma_{r e}(B) \subseteq \sigma_{e}\left(M_{C}\right)
\end{aligned}
$$

Then, $\lambda_{n} \in i s o\left(\sigma_{e}(A) \cup \sigma_{e}(B)\right) \subseteq \sigma_{e}\left(M_{C}\right), n=1,2, \ldots$ Since $\lim _{n \rightarrow \infty} \lambda_{n}=0$, so $0 \in \operatorname{acco}_{e}\left(M_{C}\right)$.

Therefore $\partial\left(a c c \sigma_{e}(A) \cup a c c \sigma_{e}(B)\right) \subseteq a c c \sigma_{e}\left(M_{C}\right)$. This proves (2).

$a c c \sigma_{e}\left(M_{C}\right) \subseteq a c c \sigma_{e}(A) \cup a c c \sigma_{e}(B)$ and (2) says that the passage from $a c c \sigma_{e}\left(M_{C}\right)$ to $a c c \sigma_{e}(A) \cup a c c \sigma_{e}(B)$ is the filling in certain of the holes in $a c c \sigma_{e}\left(M_{C}\right)$. But since $\left(a c c \sigma_{e}(A) \cup a c c \sigma_{e}(B)\right) \backslash a c c \sigma_{e}\left(M_{C}\right)$ is contained in $a c c \sigma_{e}(A) \cap a c c \sigma_{e}(B)$, it follows that the filling in certain of the holes in $\operatorname{acc} \sigma_{e}\left(M_{C}\right)$ should occur in $a c c \sigma_{e}(A) \cap \operatorname{acc} \sigma_{e}(B)$.

Corollary 2.1. Let $(A, B) \in \mathcal{B}(X) \times \mathcal{B}(Y)$. If acco $\sigma_{e}(A) \cap a c c \sigma_{e}(B)$ has no interior points, then for every $C \in \mathcal{B}(Y, X)$ we have

$$
\operatorname{acc} \sigma_{e}\left(M_{C}\right)=a c c \sigma_{e}(A) \cup a c c \sigma_{e}(B)
$$

Second main result is the following theorem.

Theorem 2.2. Let $(A, B) \in \mathcal{B}(X) \times \mathcal{B}(Y)$ and $C \in \mathcal{B}(Y, X)$. Then the following assertions are equivalent

1. $\sigma_{e}\left(M_{C}\right)=\sigma_{e}(A) \cup \sigma_{e}(B)$,

2. $\operatorname{acc}_{e}\left(M_{C}\right)=a c c \sigma_{e}(A) \cup a c c \sigma_{e}(B)$. 
Proof. First we show that $W_{e} \subseteq W$.

Indeed, if $\lambda \in W_{e}$, from theorem 1.2 , we have $\lambda \in\left(\sigma_{e}(A) \cup \sigma_{e}(B)\right) \backslash \sigma_{e}\left(M_{C}\right)$, then $\lambda \notin \sigma_{e}\left(M_{C}\right)$, hence $\lambda \notin a c c \sigma_{e}\left(M_{C}\right)$. It suffice to show that

$$
\lambda \in \operatorname{acc}_{e}(A) \cup a c c \sigma_{e}(B)=\operatorname{acc}\left(\sigma_{e}(A) \cup \sigma_{e}(B)\right)
$$

Suppose that $\lambda \notin \operatorname{acc}\left(\sigma_{e}(A) \cup \sigma_{e}(B)\right)$, since $\lambda \in \sigma_{e}(A) \cup \sigma_{e}(B)$, then

$$
\begin{aligned}
\lambda \in i s o\left(\sigma_{e}(A) \cup \sigma_{e}(B)\right) & \subseteq i s o\left(\sigma_{e}(A)\right) \cup i s o\left(\sigma_{e}(B)\right) \\
& \subseteq \partial \sigma_{e}(A) \cup \partial \sigma_{e}(B) \\
& \subseteq \sigma_{l e}(A) \cup \sigma_{r e}(B) \subseteq \sigma_{e}\left(M_{C}\right)
\end{aligned}
$$

Hence $\lambda \in \sigma_{e}\left(M_{C}\right)$, contradiction. Therefore

$$
\lambda \in\left(\operatorname{acc}_{e}(A) \cup a c c \sigma_{e}(B)\right) \backslash a c c \sigma_{e}\left(M_{C}\right)
$$

By theorem 2.1, we have $\lambda \in W$. So $W_{e} \subseteq W$.

Furthermore, $W_{e} \subseteq W$ implies that

$$
\operatorname{acc} \sigma_{e}\left(M_{C}\right)=\operatorname{acc} \sigma_{e}(A) \cup a c c \sigma_{e}(B) \Longrightarrow \sigma_{e}\left(M_{C}\right)=\sigma_{e}(A) \cup \sigma_{e}(B)
$$

Conversely, if $\sigma_{e}\left(M_{C}\right)=\sigma_{e}(A) \cup \sigma_{e}(B)$ let $\lambda \notin a c c \sigma_{e}\left(M_{C}\right)$, without loss of generality, we assume that $0 \notin a c c \sigma_{e}\left(M_{C}\right)$, then there exists $\varepsilon>0$ such that $M_{C}-\lambda$ is Fredholm for all $\lambda, 0<|\lambda|<\varepsilon$, hence $\lambda \notin \sigma_{e}\left(M_{C}\right)=$ $\sigma_{e}(A) \cup \sigma_{e}(B)$. Thus both $A-\lambda$ and $B-\lambda$ are Fredholm for every $\lambda$, $0<|\lambda|<\varepsilon$. Therefore $0 \notin a c c\left(\sigma_{e}(A)\right) \cup \operatorname{acc}\left(\sigma_{e}(B)\right)$. Since $a c c \sigma_{e}\left(M_{C}\right) \subseteq$ $\operatorname{acc}_{e}(A) \cup a c c \sigma_{e}(B)$ always holds, then $a c c \sigma_{e}\left(M_{C}\right)=a c c \sigma_{e}(A) \cup a c c \sigma_{e}(B)$.

It is immediate to check the following result.

Corollary 2.2. Let $(A, B) \in \mathcal{B}(X) \times \mathcal{B}(Y)$. If acco $\sigma_{e}(A) \cap a c c \sigma_{e}(B)$ has no interior points, then for every $C \in \mathcal{B}(Y, X)$, we have we have

$$
\sigma_{e}\left(M_{C}\right)=\sigma_{e}(A) \cup \sigma_{e}(B) \quad(* *)
$$

In particular, if either $A \in \mathcal{B}(X)$ or $B \in \mathcal{B}(Y)$ is a Riesz, then (**) holds.

Now, For $(A, B) \in \mathcal{B}(X) \times \mathcal{B}(Y)$, let $L_{A}$ (resp $R_{B}$ ) be the left (resp. right) multiplication operator given by $L_{A}(X)=A X$; $\left(\operatorname{resp} . R_{B}(X)=\right.$ $X B)$, and let $\delta_{A, B}(X)=A X-X B=L_{A}(X)-R_{B}(X)$ be the usual generalized derivation associated with $A$ and $B$. We denote by $N^{\infty}(A)=$ $\bigcup_{n \geq 1} N\left(A^{n}\right)$ the generalized kernel of $A[1]$. 
Corollary 2.3. Let $A \in \mathcal{B}(X)$ and $B \in \mathcal{B}(Y)$. If $C$ is in the closure of the set

$$
R\left(\delta_{A, B}\right)+N\left(\delta_{A, B}\right)+\bigcup_{\lambda \in \mathbf{C}} N^{\infty}\left(L_{A-\lambda}\right)+\bigcup_{\lambda \in \mathbf{C}} N^{\infty}\left(R_{B-\lambda}\right)
$$

then :

$$
\operatorname{acco}_{e}\left(M_{C}\right)=a c c \sigma_{e}(A) \cup a c c \sigma_{e}(B)
$$

Proof. If $C$ is in the closure of the set

$$
R\left(\delta_{A, B}\right)+N\left(\delta_{A, B}\right)+\bigcup_{\lambda \in \mathbf{C}} N^{\infty}\left(L_{A-\lambda}\right)+\bigcup_{\lambda \in \mathbf{C}} N^{\infty}\left(R_{B-\lambda}\right)
$$

then $\sigma_{e}\left(M_{C}\right)=\sigma_{e}(A) \cup \sigma_{e}(B)$, by [2]. From theorem 2.2, we obtain the result.

Acknowledgement: The authors thank the referees for his suggestions and comments thorough reading of the manuscript.

\section{References}

[1]P. Aiena, Semi-Fredholm operators, Perturbation theory and localized SVEP. Caracas: Ediciones IVIC, 2007.

[2]C. Benhida, E. Zerouali and H. Zguitti, "Spectra of upper triangular operator matrices", Proceedings of the American Mathematical Society, vol. 133, no. 10, pp. 3013-3021, 2005, doi: 10.1090/s0002-9939-05-07812-3.

[3]D. Djordjevic, "Perturbations of spectra of operator matrices", Journal of Operator Theory, vol. 48, no. 3, pp. 467-486, 2002. [On line]. Available: http://bit.ly/20M2xQS

[4]S. Djordević and Y. Han, "A note on Weyl's theorem for operator matrices", Proceedings of the American Mathematical Society, vol. 131, no. 8, pp. 25432548, doi: 10.1090/s0002-9939-02-06808-9.

[5]H. Du and P. Jin, "Perturbation of spectrum of $2 \times 2$ operator matrices", Proceedings of the American Mathematical Society, vol. 121, no. 3, pp.761-766, doi: 10.1090/S0002-9939-1994-1185266-2.

[6] J. Han, H. Lee and W. Lee, "Invertible completions of $2 \times 2$ upper triangular operator matrices", Proceedings of the American Mathematical Society, vol. 128, no. 01, pp. 119-124, 2000, doi: 10.1090/s0002-9939-99-04965-5. 
[7] K. Laursen and M. Neumann, An introduction to local spectral theory. (London Mathematical Society Monograph, New series, vol. 20). Oxford: Clarendon Press, 2000.

[8] E. Zerouali and H. Zguitti, "Perturbation of spectra of operator matrices and local spectral theory", Journal of Mathematical Analysis and Applications, vol. 324, no. 2, pp. 992-1005, 2006, doi: 10.1016/j.jmaa.2005. 12.065 .

[9] Y. Zhang, H. Zhong, and L. Lin, "Browder spectra and essential spectra of operator matrices", Acta Mathematica Sinica, English Series, vol. 24, no. 6, pp. 947-954, 2008, doi: 10.1007/s10114-007-6339-x. 